\title{
Correction to: Spatiotemporal plant hormone analysis from cryosections using laser microdissection-liquid chromatography-mass spectrometry
}

\author{
Kazuki Yamada $^{1} \cdot$ Miyuki Nakanowatari $^{1} \cdot$ Emi Yumoto $^{2} \cdot$ Shinobu Satoh $^{3}$ (D) Masashi Asahina $^{1,2,4}$ (D)
}

Published online: 30 November 2021

(c) The Author(s) under exclusive licence to The Botanical Society of Japan 2021

\section{Correction to: Journal of Plant Research https://doi.org/10.1007/s10265-021-01360-x}

In the original publication of the article, in Table 1, the sequence of column value of tissue "Pith" was incorrectly published as "Lower Lower Non-incised". The correct column sequence should read as "Upper Lower Non-incised".

The Table 1 is revised with the correct information.

The original article can be found online at https://doi.org/10.1007/ s10265-021-01360-x.

Masashi Asahina

asahina@nasu.bio.teikyo-u.ac.jp

1 Graduate School of Science and Engineering, Teikyo University, 1-1 Toyosatodai, Utsunomiya, Tochigi 320-8551, Japan

2 Advanced Instrumental Analysis Center, Teikyo University, 1-1 Toyosatodai, Utsunomiya, Tochigi 320-8551, Japan

3 Faculty of Life and Environmental Sciences, University of Tsukuba, 1-1-1 Tennoudai, Tsukuba, Ibaraki 305-8572, Japan

4 Department of Biosciences, Teikyo University, 1-1 Toyosatodai, Utsunomiya, Tochigi 320-8551, Japan 
Table 1 Isolated tissue from cryosection by laser microdissection $\left(\mathrm{mm}^{2}\right)$

\begin{tabular}{|c|c|c|c|c|c|c|c|c|c|}
\hline \multirow{2}{*}{$\begin{array}{l}\text { Tissue } \\
\text { Region }\end{array}$} & \multicolumn{3}{|c|}{ Epidermis/cortex } & \multicolumn{3}{|c|}{ Vascular bundle } & \multicolumn{3}{|l|}{ Pith } \\
\hline & Upper & Lower & Non-incised & Upper & Lower & Non-incised & Upper & Lower & Non-incised \\
\hline \multicolumn{10}{|l|}{ Non-incised } \\
\hline Exp.1 & - & - & 122.7 & - & - & 261.3 & - & - & 572.0 \\
\hline Exp.2 & - & - & 104.0 & - & - & 217.3 & - & - & 335.3 \\
\hline Exp.3 & - & - & 140.0 & - & - & 424.7 & - & - & 526.0 \\
\hline \multicolumn{10}{|l|}{ Incised after $1 \mathrm{~h}$} \\
\hline Exp.1 & 26.0 & 28.7 & 37.3 & 46.7 & 49.3 & 58.0 & 243.3 & 244.7 & - \\
\hline Exp.2 & 36.0 & 34.7 & 60.7 & 61.3 & 60.7 & 106.0 & 228.0 & 232.0 & - \\
\hline Exp.3 & 27.3 & 30.0 & 60.0 & 66.0 & 70.0 & 129.3 & 239.3 & 230.0 & - \\
\hline Incised after $24 \mathrm{~h}$ & & & & & & & & & - \\
\hline Exp.1 & 41.3 & 46.0 & 62.0 & 108.7 & 116.7 & 144.7 & 454.0 & 446.0 & - \\
\hline Exp.2 & 40.7 & 44.7 & 66.7 & 92.0 & 99.3 & 128.0 & 320.0 & 328.7 & - \\
\hline Exp.3 & 38.7 & 40.7 & 103.3 & 86.7 & 75.3 & 103.3 & 339.3 & 313.3 & - \\
\hline
\end{tabular}

Publisher's Note Springer Nature remains neutral with regard to jurisdictional claims in published maps and institutional affiliations. 